Supporting information for manuscript

\title{
Biofunctional silk kirigami with engineered properties
}

Sayantan Pradhan, Leonardo Ventura, Francesca Agostinacchio, Meng Xu, Ettore Barbieri, Antonella Motta, Nicola M. Pugno, Vamsi K Yadavalli *

S. Pradhan, Dr. M. Xu, Dr. V. K. Yadavalli

Department of Chemical and Life Science Engineering

Virginia Commonwealth University

601 W Main Street, Richmond VA, USA 23284

E-mail: vyadavalli@vcu.edu

F. Agostinacchio, Prof. A. Motta

BIOtech Research Center

Department of Industrial Engineering

University of Trento, TN, Italy

Dr. E. Barbieri

Japan Agency for Marine-Earth Science and Technology

Center for Mathematical Science and Advanced Technology

Computational Science and Engineering Group

3173-25, Showa-machi, Kanazawa-ku, Yokohama-city, Kanagawa, 236-0001, Japan

Prof. N. M. Pugno

Laboratory of Bio-Inspired \& Graphene Nanomechanics,

Department of Civil, Environmental and Mechanical Engineering, University of Trento, TN, Italy

$\&$

Fondazione Edoardo Amaldi, Via del Politecnico snc, Rome, Italy

L. Ventura, Prof. N. M. Pugno

School of Engineering and Materials Science,

Queen Mary University of London, L, United Kingdom

Keywords: silk fibroin, flexible kirigami, micropatterning, conducting polymer, biodegradable 
Table S1 - Examples of different materials and fabrication techniques used to form kirigami cuts.

\begin{tabular}{|c|c|c|c|c|}
\hline Materials & $\begin{array}{c}\text { Fabrication } \\
\text { method }\end{array}$ & Dimensions/shape & Applications & Ref. \\
\hline $\begin{array}{l}\text { poly(3-butylthiophene-2,5-diyl) } \\
\text { (P3BT) in poly[(9,9- } \\
\text { dioctylfluorenyl-2,7-diyl)-co-(4,4'-(N-(4- } \\
\text { sec-butylphenyl)diphenylamine)] } \\
\text { (TFB) matrix }\end{array}$ & Laser cutting & $\begin{array}{c}\text { Staggered linear cuts } \\
\text { Length of cuts } \mathrm{x} \text { the gap of horizontal } \\
\text { cuts } \mathrm{x} \text { the space of vertical staggered } \\
\text { cuts }(16 \times 3.2 \times 0.5,36 \times 2.8 \times 0.6 \\
48 \times 3.5 \times 0.9, \quad 62 \times 4.2 \times 0.6 \mathrm{~mm})\end{array}$ & $\begin{array}{c}\text { A highly stretchable, foldable, and } \\
\text { twis table conductor for light-emitting } \\
\text { diode (LED). }\end{array}$ & 1 \\
\hline $\begin{array}{l}\text { PET films coated with conductive materials } \\
\text { (ITO, Ag, PEDOT: PSS, carbon) }\end{array}$ & Laser cutting & $\begin{array}{l}\text { Staggered linear and Y-shape cuts (15 } \\
\qquad \mathrm{mm} \text { and } 5 \mathrm{~mm})\end{array}$ & $\begin{array}{c}\text { Interactive transparent wristband, 3D- } \\
\text { printed stretchable game controller, } \\
\text { textile sensor patch for joint angle } \\
\text { estimation }\end{array}$ & 2 \\
\hline Graphene oxide/PVA & $\begin{array}{l}\text { Photolithography, } \\
\text { followed by oxygen } \\
\text { plasma etching }\end{array}$ & Staggered linear cuts $(500 \mu \mathrm{m})$ & --- & 3 \\
\hline Graphite electrodes on polyimide sheet & $\mathrm{CO}_{2}$ laser & Staggered linear cuts (over $3 \mathrm{~cm}$ ) & Stretchable supercapacitor & 4 \\
\hline Gold nanofilms & $\begin{array}{l}\text { Dual-beam focused } \\
\text { ion beam } \\
\text { (FIB)/SEM system }\end{array}$ & Arcs and 3D microdome (sub-50-nm) & 3D optical chirality & 5 \\
\hline $\begin{array}{l}\text { Polycrystalline monolayer graphene grown } \\
\text { on copper }\end{array}$ & Optical lithography & $\begin{array}{c}\text { In plane kirigami springs, out of plane } \\
\text { pyramidal spring }\end{array}$ & $\begin{array}{c}\text { Conductivity of the springs and } \\
\text { environment responsive actuation of } \\
\text { out of plane kirigami structure }\end{array}$ & 6 \\
\hline Thin film gallium arsenide & $\begin{array}{l}\text { Photolithography } \\
\text { and chemical } \\
\text { etching }\end{array}$ & Staggered linear cuts & $\begin{array}{c}\text { Thin-film GaAs solar cells and a } \\
\text { kirigami tracker made from Kapton } \\
\text { sheet }\end{array}$ & 7 \\
\hline $\begin{array}{l}\text { PET and PDMS films with PEDOT:PSS and } \\
\text { CNT }\end{array}$ & Laser cutting & Staggered lines & $\begin{array}{l}\text { Wearable health monitoring device } \\
\text { with temperature, ECG, UV and a } 3 \\
\text { axis acceleration sensor }\end{array}$ & 8 \\
\hline Gold traces embedded in Parylene C & $\begin{array}{l}\text { Oxygen plasma } \\
\text { etching }\end{array}$ & Serpentine cuts & Flexible strain sens or & \\
\hline $\begin{array}{llll}\text { Mono-layer molybdenum } & \text { disulfide } & \text { on } \\
\text { PDMS } & & \\
\end{array}$ & $\begin{array}{c}\text { Molding and } \\
\text { plasma etching }\end{array}$ & $\begin{array}{c}\text { Linear patterns of kirigami springs, } \\
\text { kirigami pyramids, and out-of-plane } \\
\text { kirigami springs with alternating C- } \\
\text { shapes }\end{array}$ & Strain sensor & 10 \\
\hline Graphene oxide- silk fibroin biocomposite & Drag knife cutting & $\begin{array}{l}\text { Staggered lines, pop-up box, spiral } \\
\text { slinky, spinner, parallel lines }\end{array}$ & $\begin{array}{c}\text { Energy harvester/ triboelectric } \\
\text { nanogenerator }\end{array}$ & $\pi$ \\
\hline $\begin{array}{lllll}\mathrm{LiFePO}_{4} & \text { (LFP) } & \text { or } & \mathrm{Li}_{4} \mathrm{Ti}_{5} \mathrm{O}_{12} & \text { (LTO), }\end{array}$ & Template printing & Staggered lines & Stretchable battery & 12 \\
\hline
\end{tabular}




\begin{tabular}{|c|c|c|c|c|}
\hline $\begin{array}{l}\text { poly(vinylidene fluoride) (PVDF), and } \\
\text { multiwalled carbon nanotubes (MWCNTs; } \\
\text { conductive additive) mixed in N-methyl-2- } \\
\text { pyrrolidone (NMP) }\end{array}$ & & & & \\
\hline $\begin{array}{l}\text { Paper and pre-strained shape memory } \\
\text { polymer-based shrink paper }\end{array}$ & Laser cutting & Square and triangle cuts & $\begin{array}{c}\text { Programmable } 3 \mathrm{D} \text { self-folding } \\
\text { kirigami machines and soft turning } \\
\text { robots }\end{array}$ & 13 \\
\hline $\begin{array}{l}\text { Super-stretchable abrasion resistant natural } \\
\text { rubber, PDMS }\end{array}$ & $\begin{array}{l}\text { Laser cutting, } \\
\text { molding }\end{array}$ & Perpendicular lines & $\begin{array}{l}\text { Shape-morphing mechanical } \\
\text { metamaterials }\end{array}$ & 14 \\
\hline
\end{tabular}



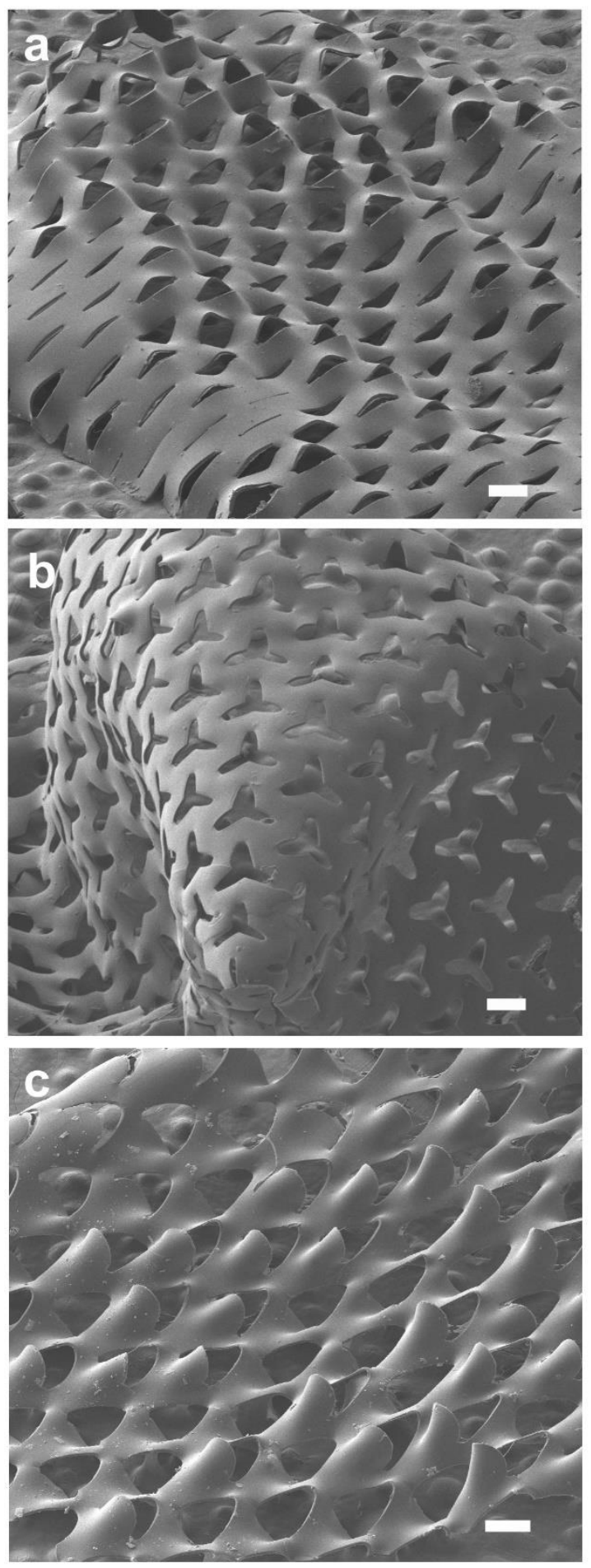
Figure S1 - Scanning Electron Microscopy (SEM) images of large area microscale silk kirigami films with different pattern geometries (top to bottom - (a) slits, (b) Y-cuts, and (c) saddle shapes). 


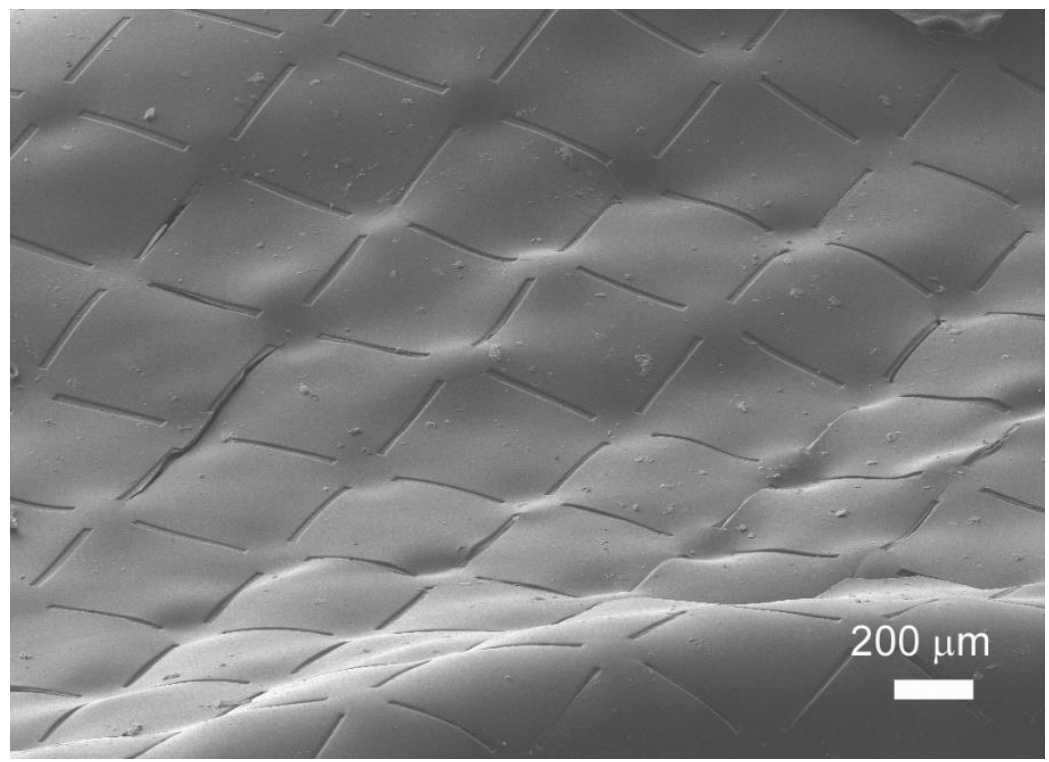

Figure S2 -By reducing the exposure time (in this experiment, the exposure time was $1 \mathrm{~s}$ ), it is possible to engineer weak spots in the films in the form of unopened or partial cuts. This technique may be used to photolithographically form wrinkles in films. 


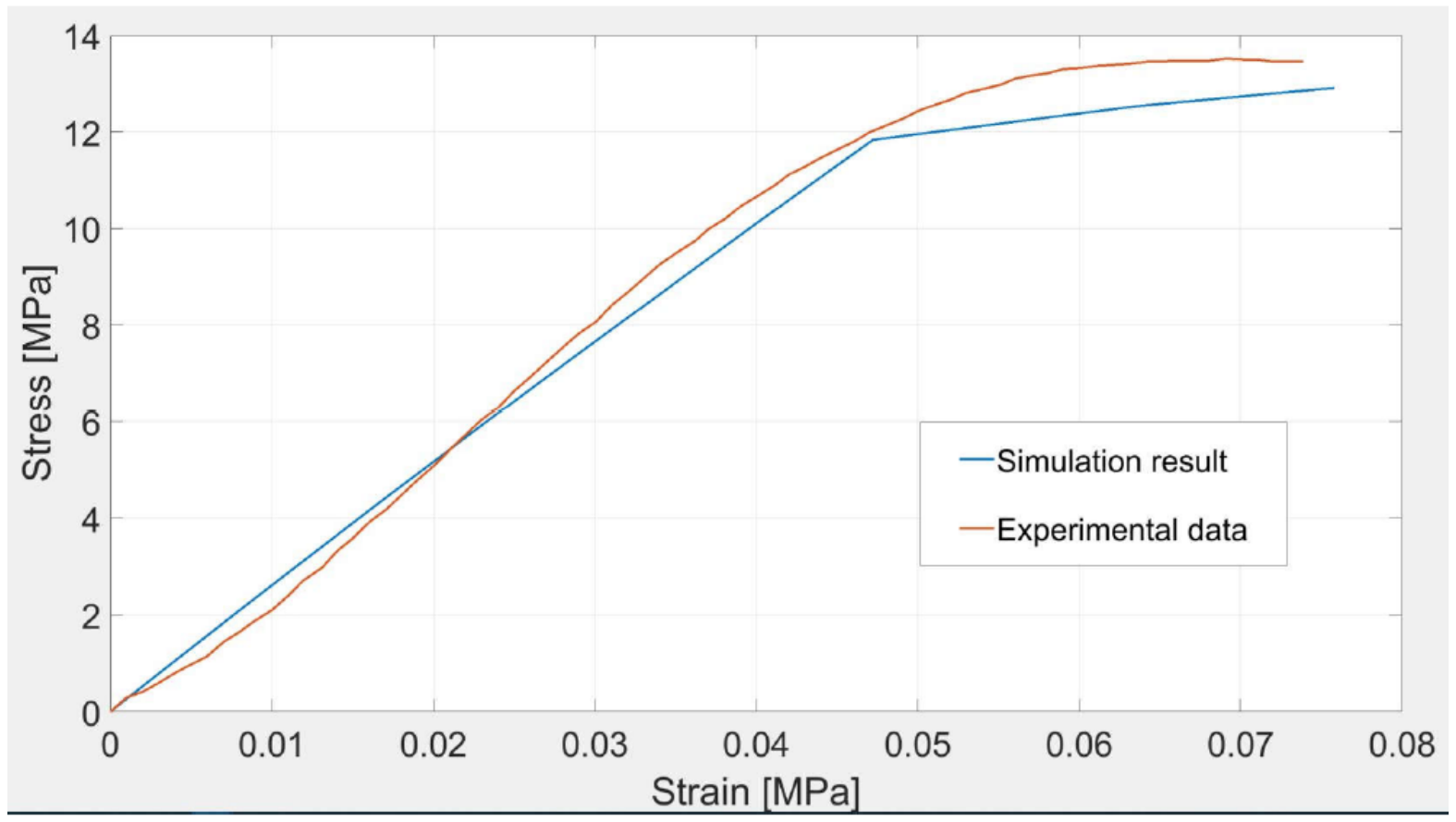

Simulation and comparison to a pristine film (no cuts).

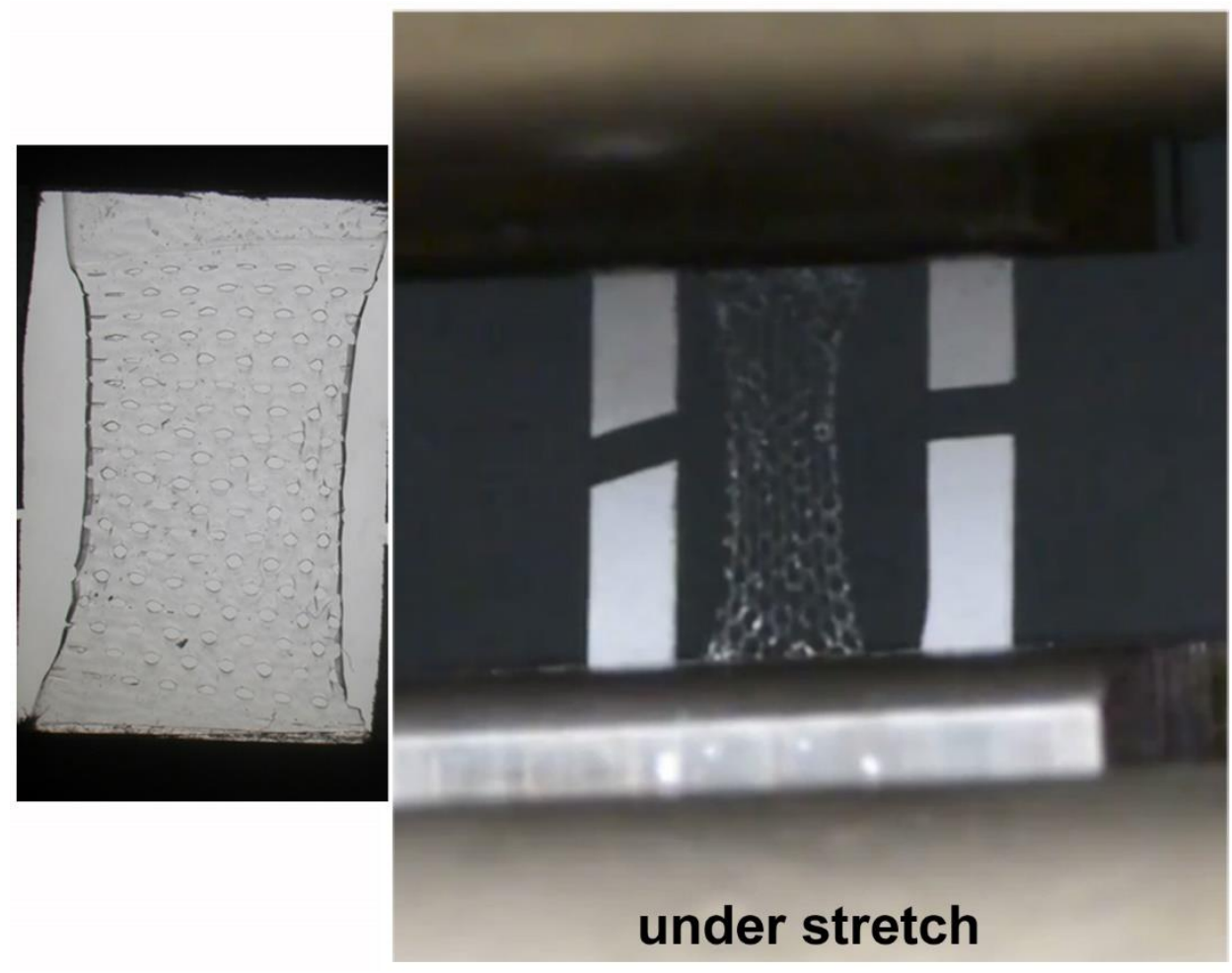

Figure S3 - Tensile testing of the kirigami films using a conventional tester. The films can be stretched to $40 \%$ strain without failure. In comparison, pristine films fail at 5-8\% strain. 

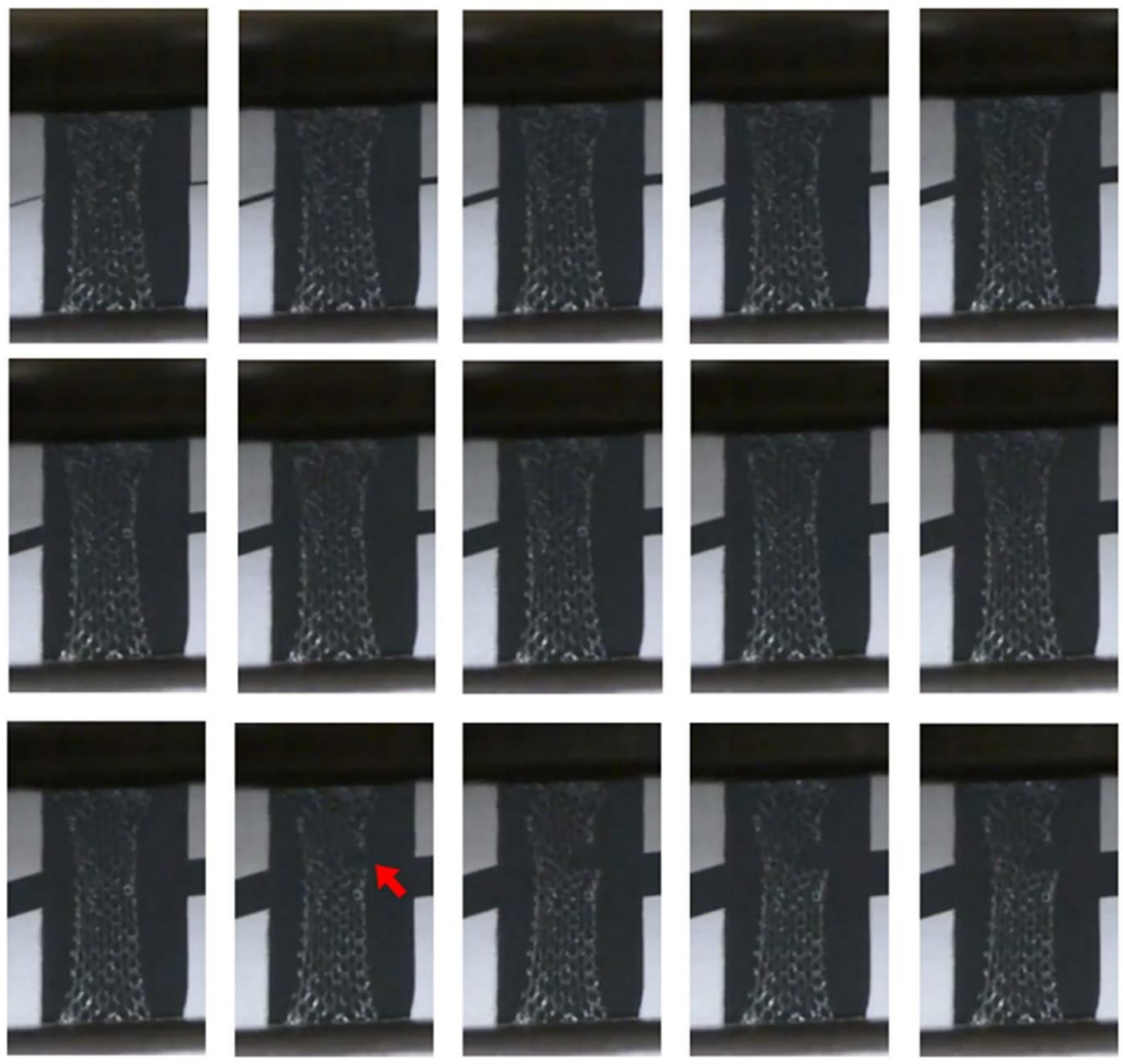

Figure S4 - Stills from video taken for kirigami stretch. The red-arrow in the bottom row indicates the point of failure of the film. The failure then propagates through the entire film. The tensile apparatus retreats prior to complete film breakage. 


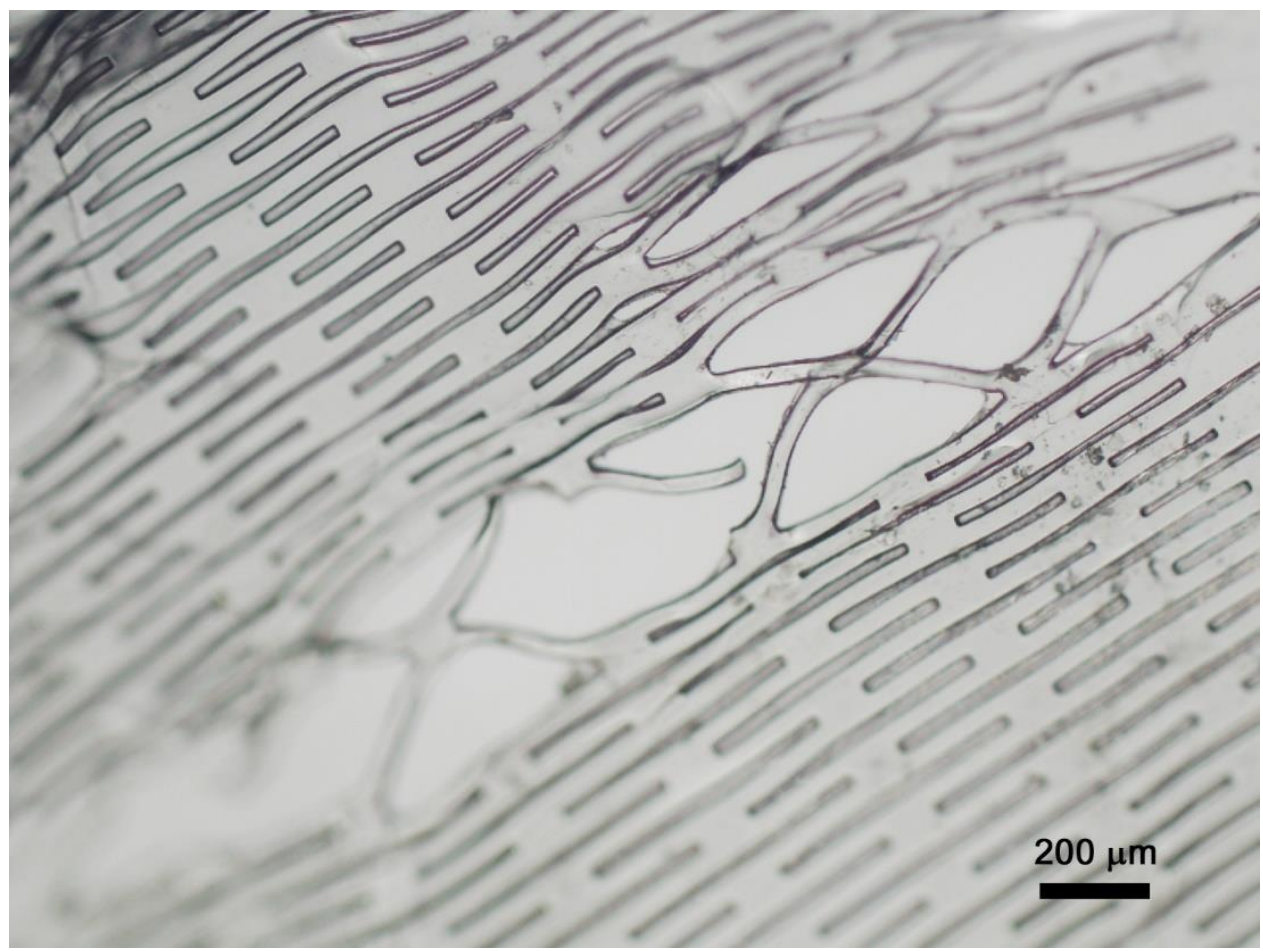

Figure S5 - Optical image showing one of the modes of failure on stretching a silk kirigami film with a slit geometry. Even though some of the cuts may tear, the entire structure does not come apart. 


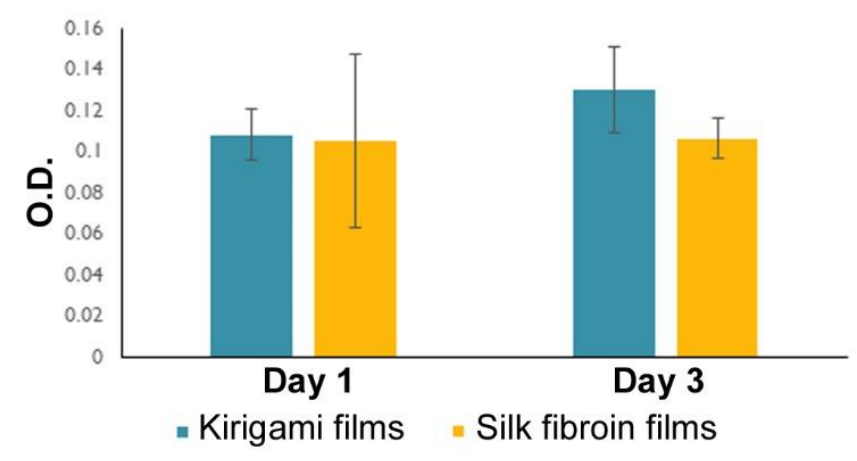

Figure S6: Cell viability. An MTT ((3-(4,5-Dimethylthiazol-2-yl)-2,5-diphenyltetrazolium bromide, a tetrazole) assay was conducted to determine the cell activity and viability. Human fibroblast cells were used and at least 4 replicates of each sample were measured. Following cell culture for $24 \mathrm{~h}$, the cells were washed with $0.5 \mathrm{ml}$ PBS and removed from the wash solution. 50 ul of MTT reagent (Abcam) were added and incubated at $37^{\circ} \mathrm{C}$ for $3 \mathrm{hrs}$. After MTT Incubation, $150 \mu \mathrm{l}$ of MTT solvent was added to elute the purple ice crystals of MTT and shaken for 15 min using an orbital shaker. $1.5 \mathrm{ml}$ of DMSO was added to completely dissolve purple formazan. The absorbance was measured at $570 \mathrm{~nm}$ using a microplate reader and reported as O.D. in the figure. It can be seen that the presence of kirigami cuts did not negatively affect cell viability in comparison with standard fibroin film in the culture period considered. Note that the cell viability of silk fibroin films via MTT assays has been reported by other groups. ${ }^{15-16}$ 


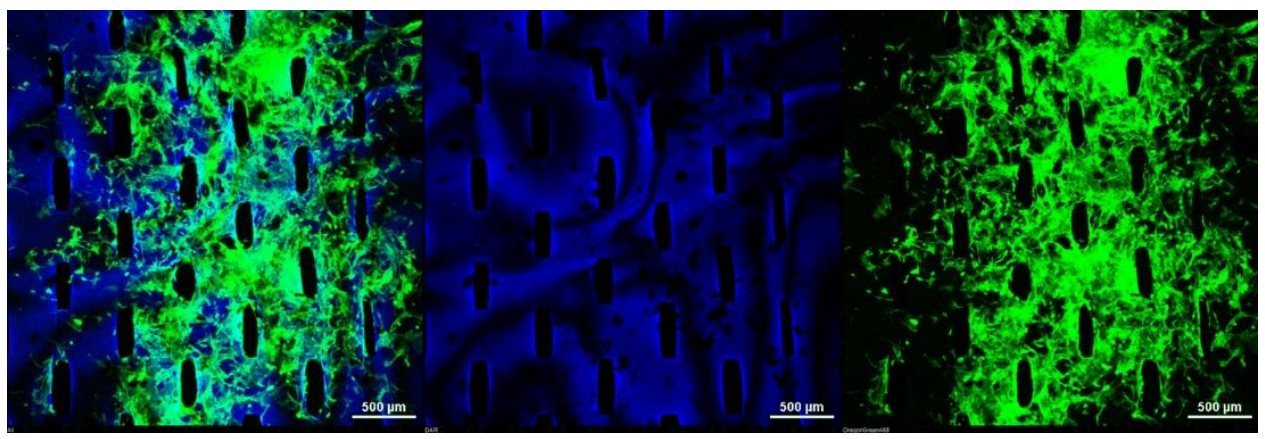

Attachment of C2C12 cells on the kirigami films preconditioned with expansion media.

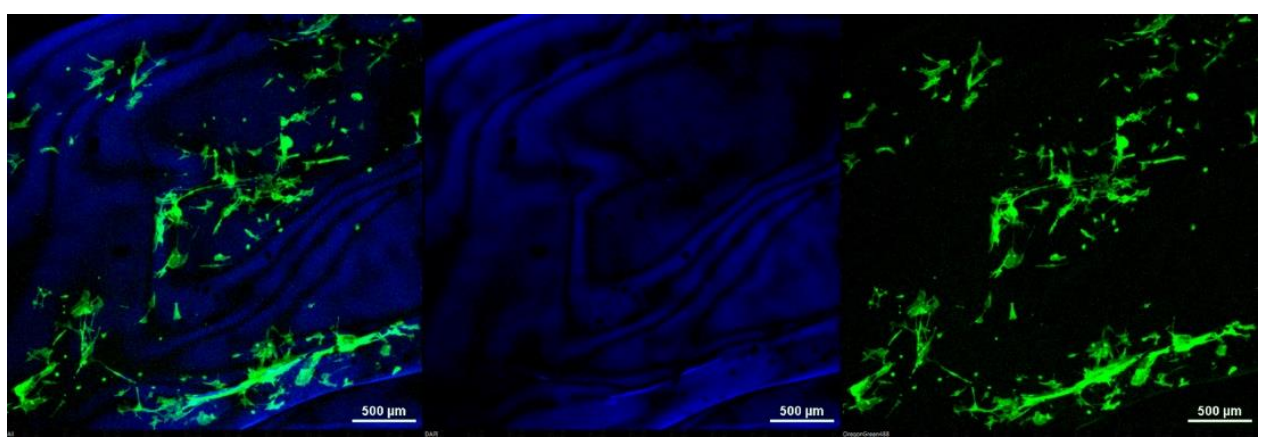

Control film (with no cuts) preconditioned with expansion media.

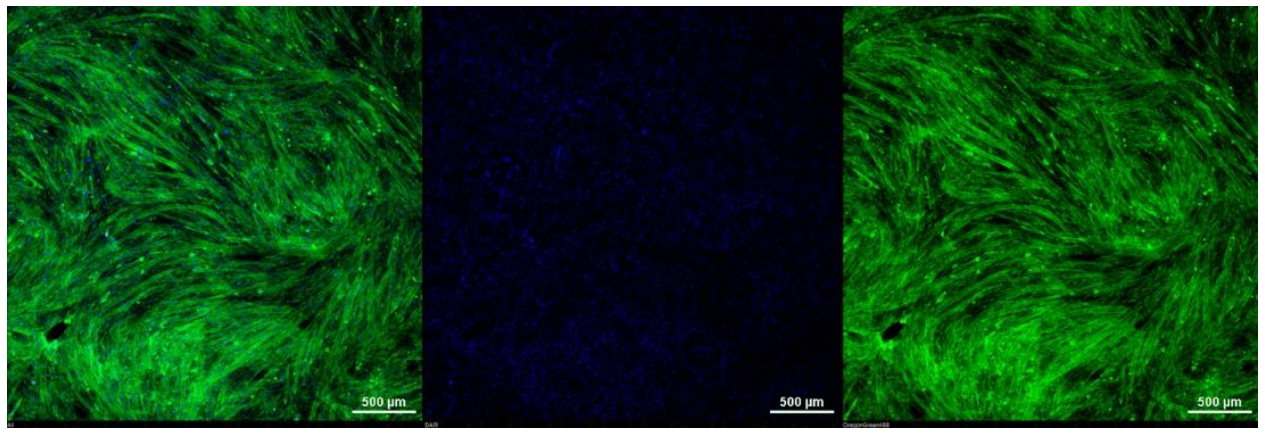

TCP control

Figure S7: Cell culture experiments on the silk kirigami films showing a) biocompatibility of the films, their robustness to cell sterilization protocols and b) the spreading of cells on the films. 


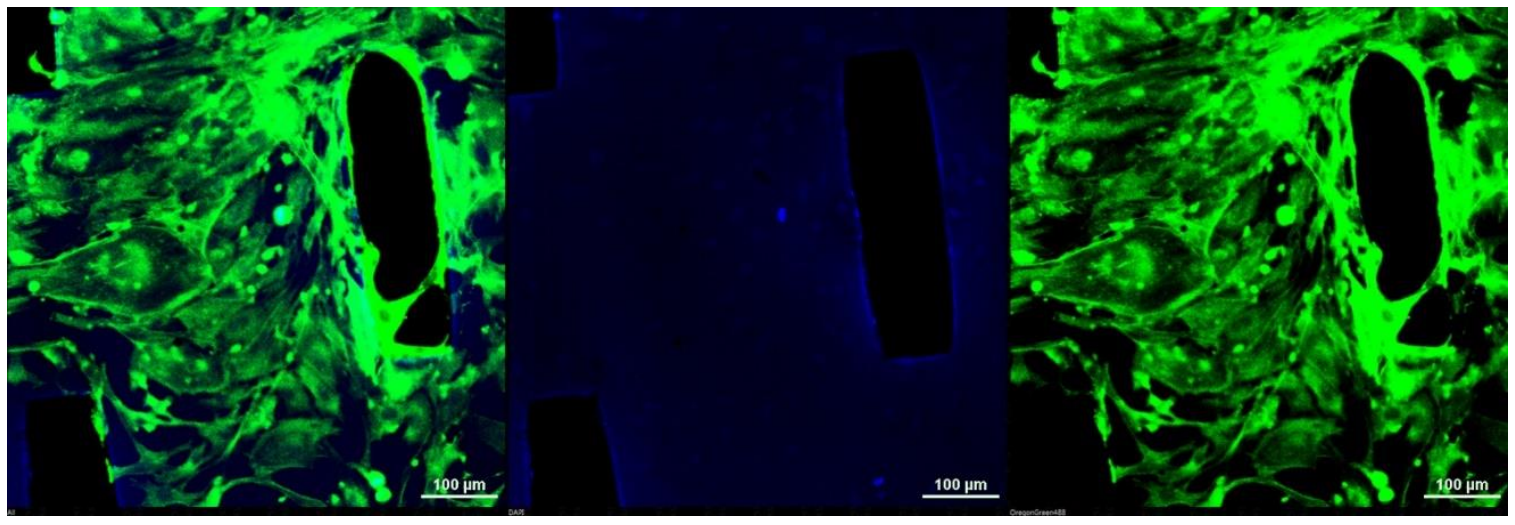

Figure S8 - Zoomed in image of a silk kirigami film that was pre-treated with fibronectin prior to attachment of $\mathrm{C} 2 \mathrm{C} 12$ cells. Scale bar $=100 \mu \mathrm{m}$. 

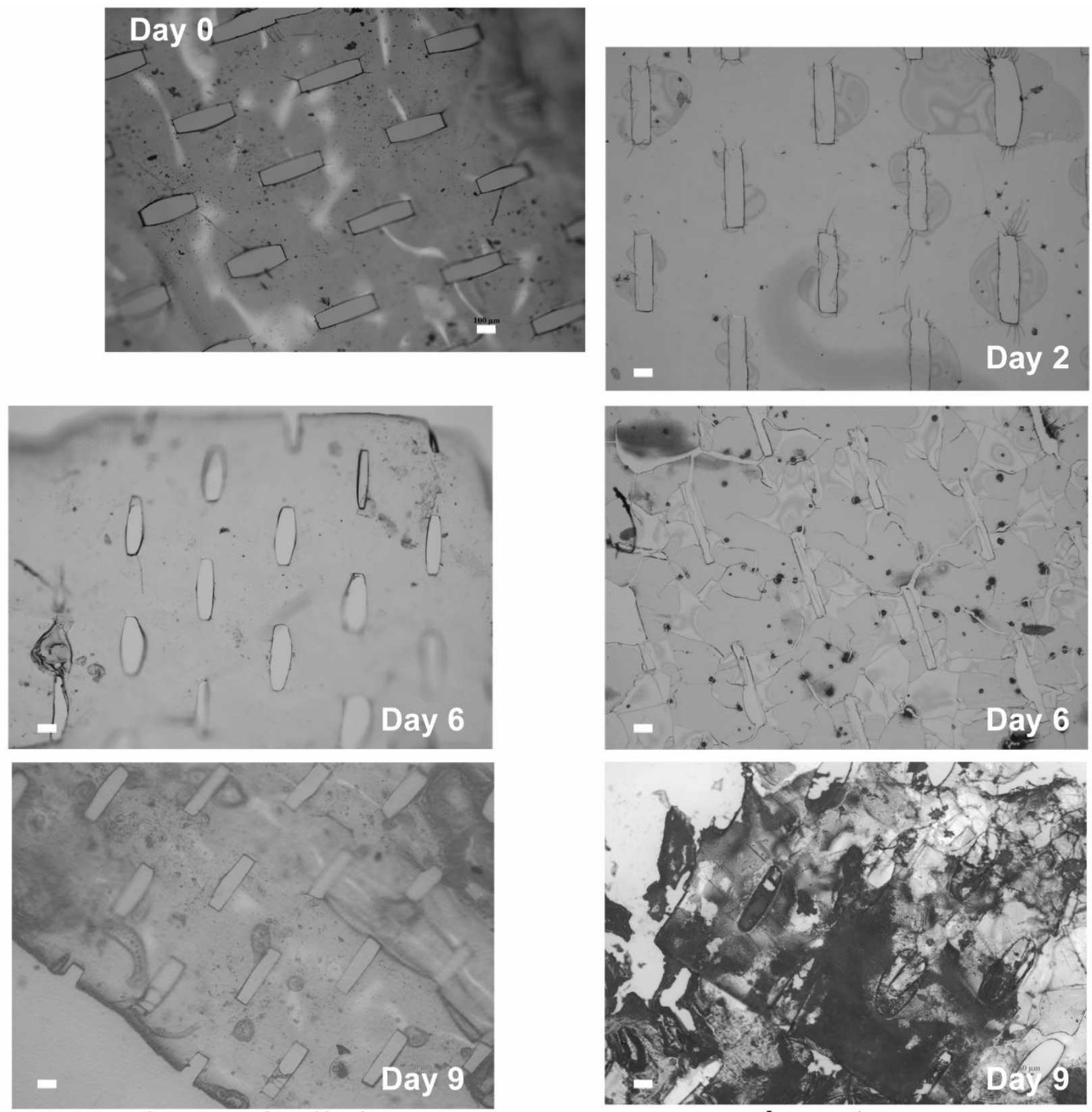

Control (buffer)

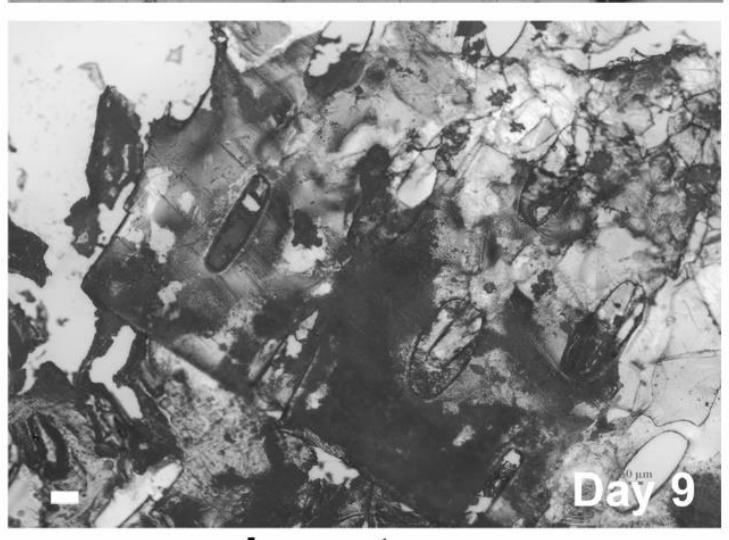

In protease

Figure S9 - Proteolytic degradation of silk kirigami sheets. On the left, films in (control) PBS buffer remain stable over several days (stored for a month with no loss in properties or morphology). On the right, a thin film $(\sim 10 \mu \mathrm{m})$ is completely biodegraded in solution of proteolytic enzyme (protease XIV), showing for the first time, a kirigami film that can be degraded. By controlling the thickness of the films, the rate of degradation can be controlled. Scale bar $=100 \mu \mathrm{m}$. 

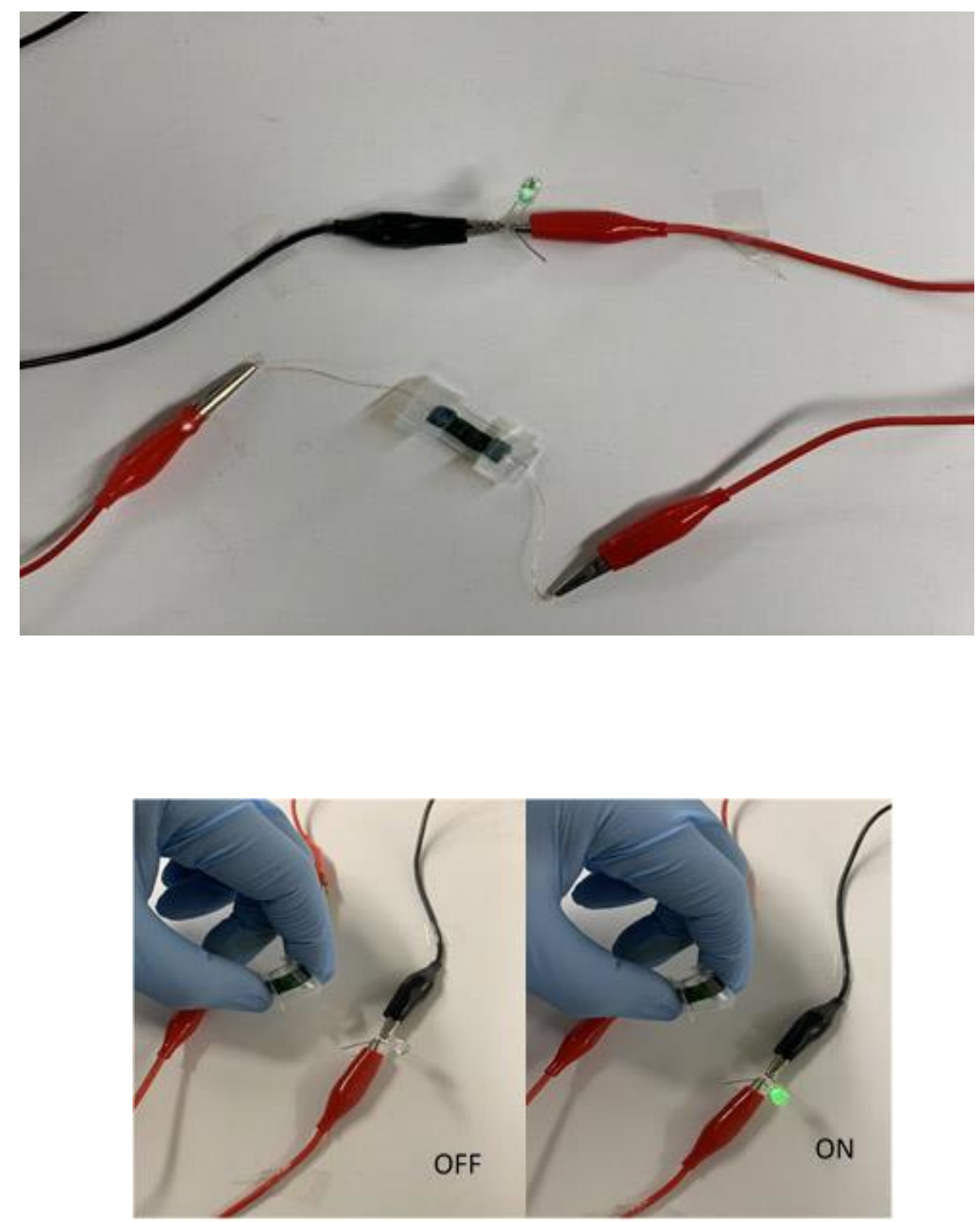

Figure S10 - Experimental set up to measure the properties of the silk kirigami films (11\% PANI) under different bending conditions. The fibroin films containing PANI were developed in $1 \mathrm{M} \mathrm{LiCl} / \mathrm{DMSO}$, and washed. The film was attached to a PDMS slab and wires were connected using carbon paste and epoxy. $100 \mu \mathrm{L}$ of PBS was dropcast on the films. Following a 5-minute incubation, the buffer was pipetted out. The film was subjected to bends of various radius of curvature and cyclic voltammetry was performed from $-1 \mathrm{~V}$ to $1 \mathrm{~V}$ (results presented from $-0.5 \mathrm{~V}$ to $0.5 \mathrm{~V})$. 


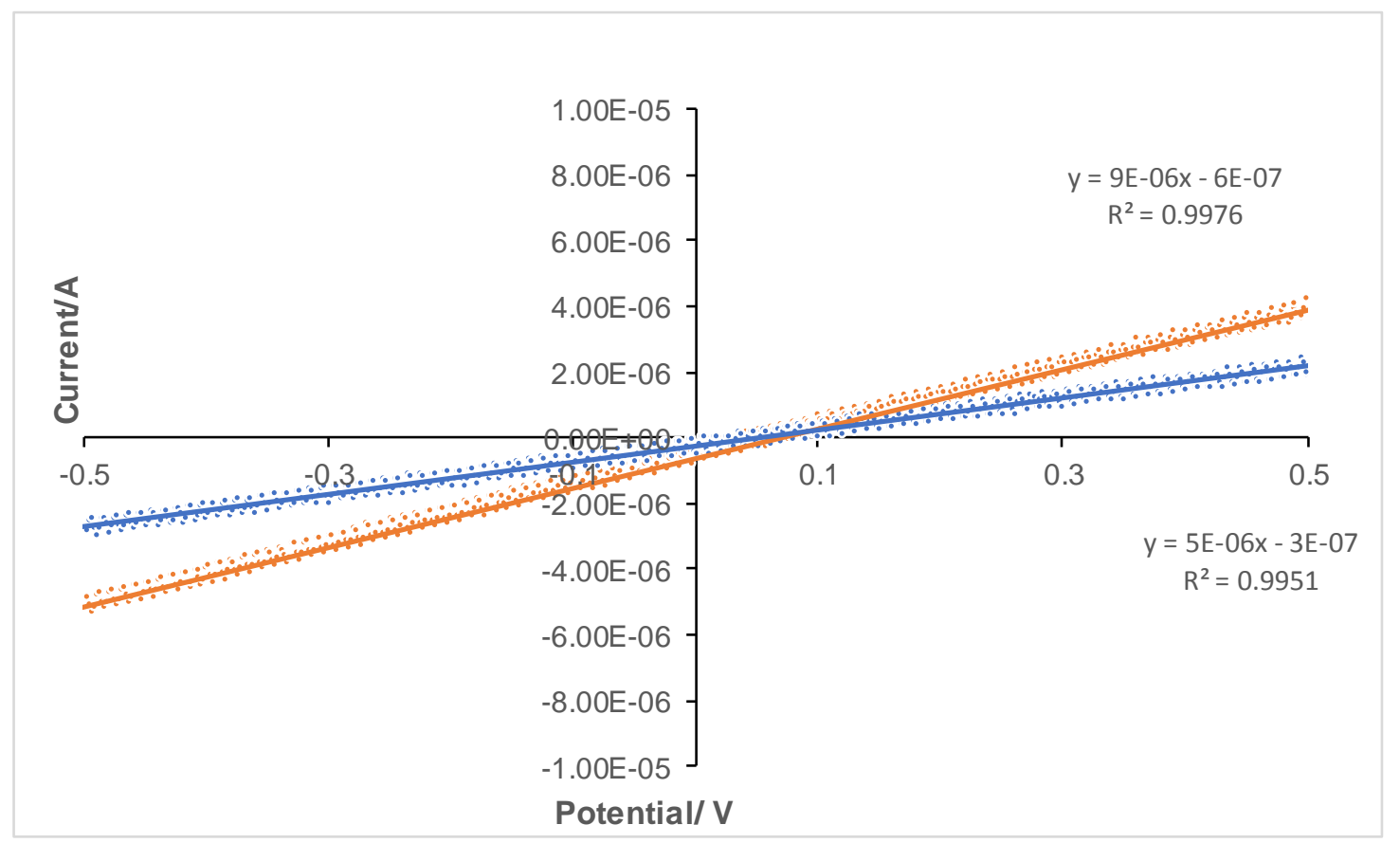

Figure S11 - Linear sweep voltammetry was taken under relaxed and stretched conditions. Films with $100 \mu \mathrm{m}$ cuts (slit geometry - slit width $100 \mu \mathrm{m}$, length $500 \mu \mathrm{m}$, gap spacing - 500 $\mu \mathrm{m}$ and gap width $500 \mu \mathrm{m}$ ) unstretched (orange line) and with 10\% linear strain (stretch - blue line). Conductance changes from $9 \mu \mathrm{S}$ to $5 \mu \mathrm{S}$. 


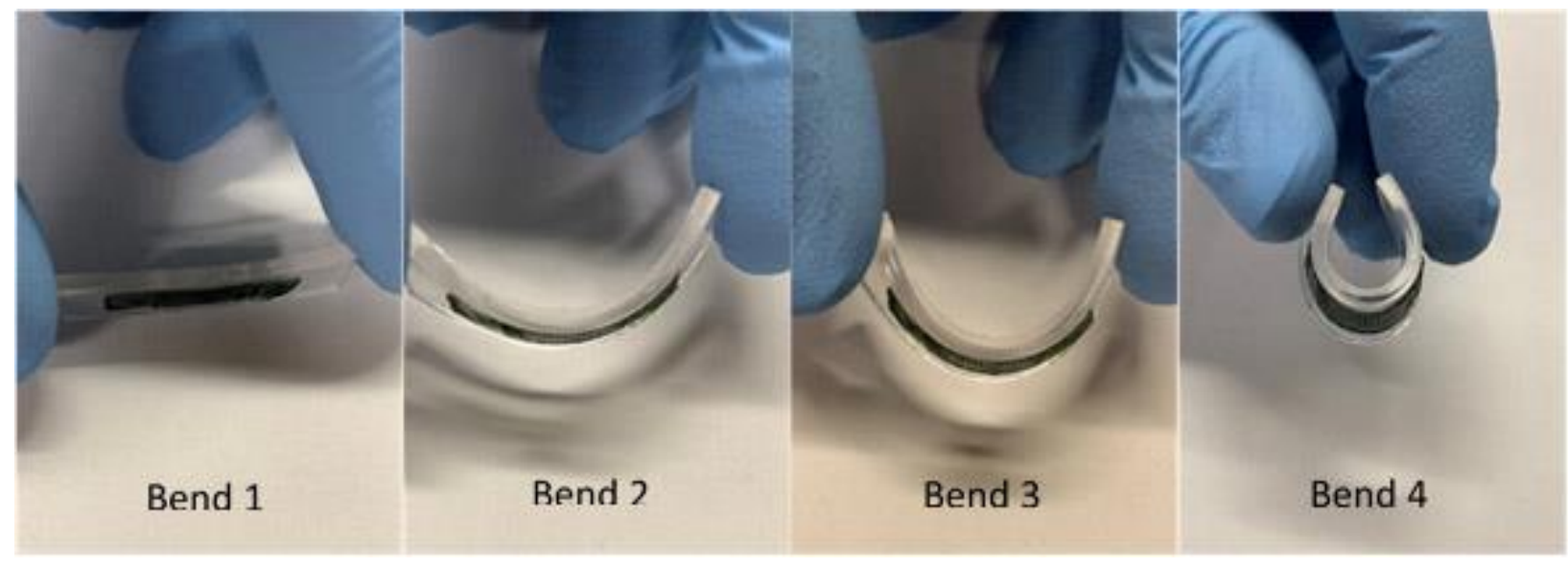

Figure S12 - Bending experiment corresponding to the data shown in Figure 4 of the manuscript. Bend 2 is $20^{\circ}$, Bend 3 is $55^{\circ}$ (knee), Bend 4 is $180^{\circ}$. 


\section{Theoretical model for the strength of silk kirigami films}

\begin{tabular}{|l|l|l|l|l|}
\hline $\mathbf{2}$ & $\mathbf{1 . 2 5}$ & $\mathbf{1 . 6 7}$ & $\mathbf{2 . 5 0}$ & $+\infty$ \\
\hline $\mathbf{2}$ & & & & \\
\hline $\mathbf{4}$ & 0.4539 & 0.4174 & 0.2649 & 0.2030 \\
\hline $\mathbf{6}$ & 0.1953 & 0.2408 & 0.1764 & 0.0743 \\
\hline $\mathbf{8}$ & 0.0350 & 0.1182 & 0.1181 & 0.0349 \\
\hline $\mathbf{1 0}$ & -0.0953 & 0.0421 & 0.0735 & 0.0170 \\
\hline$+\infty$ & -0.1773 & -0.0034 & 0.0508 & 0.0130 \\
\hline
\end{tabular}

Table S2: Theoretical shielding factors for different kirigami patterns.

\section{Simulation details:}

The commercial software ABAQUS was used to compute the deformations of the kirigami films for three patterns (500 $\mu \mathrm{m}$ cuts, $1000 \mu \mathrm{m}$ cuts and ' $\mathrm{Y}$ '-shaped or branched cuts). ${ }^{17}$ The Python script contains three sections: mechanical and geometrical parameters, cut patterns and the constitutive model. The section "Mechanical and geometrical parameters" shows the units used in the model, and the values of the parameters used to perform a specific simulation: geometric parameters, mechanical properties of the material [Young's modulus E, Poisson's ratio v], mass density, and the loading condition of the sample (load or defect).

The geometry consists of three different parts, called "sketches" in ABAQUS. The "plateSketch" defines the part shape and dimensions, the "cutSketch" contains the pattern of the cuts, and the "partSketch" creates a domain partition to control the meshing algorithm. The mesh is composed of 6-node triangular thin shell elements (STRI65) near the cut tips to limit elements distortion upon cut opening, and 8-node quadrangular thin shells (S8R5) for the rest of the domain. To define the boundary conditions, we used a sub-set called "fixedEdge" containing the upper shell edge, whereas "movingEdge" contains the lower shell edge. The script proceeds to create cuts and partitions. The function "cut" takes the central point of the cut and cut type (i.e. horizontal, vertical or curvilinear) as input and adds the cut line to the "cutSketch" and four circles centered on the two cut tips (two each) to the "partSketch". Moreover, partitioning each cut in two halves 
allows introducing a "defect" in correspondence of the middle point of each cut. This defect triggers the out-of-plane instability typical observed in kirigami structures.

The mechanical constitutive model is elastoplastic with isotropic linear elasticity and linear hardening. The material density is necessary since the buckling onset requires a dynamic analysis.

Finally, the "part" object collects all the geometrical and material parameters. This is translated in a physical domain by defining an "Instance" of "Assembly" in the ABAQUS language. Boundary conditions were set. The sub-domain "fixedEdge" is constrained, removing six degrees of freedom. During the first part of the simulation, the "movingEdge" was fixed, and one boundary condition for this subdomain was added.

Videos of the Simulations are included as separate files. 


\section{References:}

1. Guan, Y.-S.; Zhang, Z.; Tang, Y.; Yin, J.; Ren, S., Kirigami-Inspired Nanoconfined Polymer Conducting Nanosheets with 2000\% Stretchability. Adv. Mater. 2018, 30, 1706390.

2. Groeger, D.; \#252; Steimle, r., LASEC: Instant Fabrication of Stretchable Circuits Using a Laser Cutter. In Proceedings of the 2019 CHI Conference on Human Factors in Computing Systems, ACM: Glasgow, Scotland UK, 2019;1-14.

3. Shyu, T. C.; Damasceno, P. F.; Dodd, P. M.; Lamoureux, A.; Xu, L.; Shlian, M.; Shtein, M.; Glotzer, S. C.; Kotov, N. A., A Kirigami Approach to Engineering Elasticity in Nanocomposites through Patterned Defects. Nat. Mater. 2015, 14, 785.

4. $\mathrm{Xu}, \mathrm{R} . ;$ Zverev, A.; Hung, A.; Shen, C.; Irie, L.; Ding, G.; Whitmeyer, M.; Ren, L.; Griffin, B.; Melcher, J.; Zheng, L.; Zang, X.; Sanghadasa, M.; Lin, L., Kirigami-inspired, Highly Stretchable Micro-supercapacitor Patches Fabricated by Laser Conversion and Cutting. Microsyst. Nanoeng. 2018, 4, 36.

5. Liu, Z.; Du, H.; Li, J.; Lu, L.; Li, Z.-Y.; Fang, N. X., Nano-kirigami with giant optical chirality. Sci. Adv. 2018, 4, eaat4436.

6. Blees, M. K.; Barnard, A. W.; Rose, P. A.; Roberts, S. P.; McGill, K. L.; Huang, P. Y.; Ruyack, A. R.; Kevek, J. W.; Kobrin, B.; Muller, D. A.; McEuen, P. L., Graphene kirigami. Nature 2015, 524, 204.

7. Lamoureux, A.; Lee, K.; Shlian, M.; Forrest, S. R.; Shtein, M., Dynamic Kirigami Structures for Integrated Solar Tracking. Nat. Commun. 2015, 6.

8. Yamamoto, Y.; Harada, S.; Yamamoto, D.; Honda, W.; Arie, T.; Akita, S.; Takei, K., Printed Multifunctional Flexible Device with an Integrated Motion Sensor for Health Care Monitoring. Sci. Adv. 2016, 2, e1601473.

9. Baldwin, A.; Meng, E., Kirigami Strain Sensors Microfabricated From Thin-Film Parylene C. J. Microelectromech. Syst. 2018, 27, 1082-1088.

10. Zheng, W.; Huang, W.; Gao, F.; Yang, H.; Dai, M.; Liu, G.; Yang, B.; Zhang, J.; Fu, Y. Q.; Chen, X.; Qiu, Y.; Jia, D.; Zhou, Y.; Hu, P., Kirigami-Inspired Highly Stretchable Nanoscale Devices Using Multidimensional Deformation of Monolayer MoS2. Chem. Mater. 2018, 30, 6063-6070.

11. Ma, R.; Wu, C.; Wang, Z. L.; Tsukruk, V. V., Pop-Up Conducting Large-Area Biographene Kirigami. ACS Nano 2018, 12, 9714-9720. 
12. Bao, Y.; Hong, G.; Chen, Y.; Chen, J.; Chen, H.; Song, W.-L.; Fang, D., Customized Kirigami Electrodes for Flexible and Deformable Lithium-Ion Batteries. ACS Appl. Mater. Interfaces 2020, 12, 780-788.

13. Tang, Y.; Li, Y.; Hong, Y.; Yang, S.; Yin, J., Programmable Active Kirigami Metasheets with more Freedom of Actuation. Proc. Natl. Acad. Sci. U.S.A. 2019, 116, 26407.

14. Choi, G. P. T.; Dudte, L. H.; Mahadevan, L., Programming Shape using Kirigami Tessellations. Nat. Mater. 2019, 18, 999-1004.

15. Liu, T.-1.; Miao, J.-c.; Sheng, W.-h.; Xie, Y.-f.; Huang, Q.; Shan, Y.-b.; Yang, J.-c., Cytocompatibility of Regenerated Silk Fibroin Film: A Medical Biomaterial applicable to Wound Healing. Journal of Zhejiang University SCIENCE B 2010, 11, 10-16.

16. Panico, A.; Paladini, F.; Pollini, M., Development of Regenerative and Flexible Fibroinbased Wound Dressings. J. Biomed. Mater. Res. Part B 2019, 107, 7-18.

17. Barbieri, E.; Ongaro, F.; Pugno, N. M., A J-Integral-Based Arc-Length Solver for Brittle

and Ductile Crack Propagation in Finite Deformation-Finite Strain Hyperelastic Solids with an application to Graphene Kirigami. Computer Methods in Applied Mechanics and Engineering 2017, 315, 713-743. 